\title{
INTELIGENCIAS MÚLTIPLES: CARACTERÍSTICA CONGRUENTE DEL LIDERAZGO ÉTICO EN ENTIDADES FINANCIERAS
}

\author{
MULTIPLE INTELLIGENCE: ETHICAL LEADERSHIP FEATURE CONSISTENT \\ FINANCIAL INSTITUTIONS
}

\author{
Diamela Nava*, Cristina Seijo** y Cristina Mier***
}

\begin{abstract}
RESUMEN
Este estudio tiene como propósito realizar un contraste teórico que sustenta el análisis sobre las Inteligencias múltiples: característica congruente del Liderazgo ético en Entidades Financieras. No obstante, esta investigación se realizó bajo un enfoque cualitativo, de tipo descriptiva, mediante el análisis documental, donde finalmente se pudo considerar que las inteligencias múltiples servirían de apoyo para poner en práctica ciertas capacidades, para el logro de los objetivos con la finalidad y desde el punto de vista racional, saber cómo establecer cambios significativos en algunos aspectos, es decir, al modo de evaluar las capacidades cognitivas del talento humano que integra en las organizaciones. Por tanto, el papel del líder es guiar y apoyar el desarrollo del potencial humano de su grupo de interés como una comunidad con el fin de lograr las aspiraciones de la organización utilizando la inteligencia como herramienta estratégica en distintas maneras para no limitar su imaginación, juicio y acción cooperativa.
\end{abstract}

Palabras Clave: Actuación humana, Entidades financieras, Inteligencias múltiples, Liderazgo ético.

\begin{abstract}
This study aims to make a theoretical underpinning contrast analysis on the multiple intelligences: consistent feature of Ethical Leadership in Financial Institutions. However, this research was conducted under a qualitative approach, a descriptive, using document analysis, which eventually might be considered that would support multiple intelligences to implement certain capabilities, to achieve the objectives with the purpose and from the rational point of view, to know how to establish significant changes in some ways it is, the way to assess the cognitive abilities of integrating human talent in organizations. Therefore, the role of the leader is to guide and support the development of human potential in their group as a community of interest in order to achieve the aspirations of the organization using intelligence as a strategic tool in different ways to not limit your imagination, judgment, and cooperative action.
\end{abstract}

Keywords: Multiple intelligences, ethical leadership, human performance, financial institutions.

\footnotetext{
Fecha de recepción: Abril 22 de 2014 / Fecha de aceptación: Agosto 15 de 2014

Tipología: Artículo de Reflexión

Para citar este artículo: Nava, D., Seijo, C., \& Mier, C. (2014). Inteligencias múltiples: característica congruente del liderazgo ético en entidades financieras, Praxis, Vol. 10, 91 - 99
}

\footnotetext{
*Doctora en Ciencias Gerenciales Universidad Rafael Belloso Chacín, Venezuela. E-mail: nava-diamela@hotmail.com.

**Postdoctora en Gerencia de las organizaciones y Gerencia en la educación superior Universidad Rafael Belloso Chacín, Venezuela. E-mail: cristinaseijoa@hotmail.com.

***Estudiante de medicina, Investigadora activa Universidad del Zulia., Venezuela E-mail: cristinaseijoa@gmail.com.
} 


\section{INTRODUCCIÒN}

$\mathrm{E}$ n la actual sociedad, se exige que las organizaciones cuenten con una visión estratégica basada en el conocimiento de su gente, lo que conduce a pensar que el aprendizaje organizacional es una fuente de ventaja competitiva lo cual conlleva a que las empresas como organismos inteligentes influyan en la cultura y los valores de la sociedad como fundamentos axiológicos para enfrentar con éxito los retos que se plantean en el entorno. Cabe destacar que es importante la necesidad de promover las inteligencias múltiples tomando como base fundamental el liderazgo ético principalmente en la actuación humana del líder de esta manera; al proyectar una actitud congruente con el equipo de trabajo a través de la confianza se logra de manera satisfactoria una armonía transparente y auténtica en la organización.

Asimismo, poner en práctica las distintas capacidades a través de las inteligencias múltiples es generar sinergias positivas, que permitan combinar los esfuerzos de todos, con la finalidad de lograr los fines organizacionales, por tanto, el éxito empresarial estará garantizado a futuro.

De este modo, es preciso aceptar la realidad y en virtud de ello, implica que el líder puede ser inteligente sin ser moral, creativo pero no ético, ser sensible a las emociones y no usar esa sensibilidad al servicio de los demás, porque puede interpretarse como no ejercer la ética en el liderazgo. Sin embargo, se debe aceptar y reconocer la verdad pero a la vez mantenerse ciego frente a la belleza y la bondad.

\section{Inteligencias múltiples: un proceso de ingeniosidad y capacidad cognitiva en el ser humano}

Según los postulados de Beauport \& Díaz (2008), las inteligencias múltiples consisten en la capacidad para realizar innovaciones valiosas que permiten enriquecer el comportamiento adaptativo para los conocimientos diferenciadores de las potencialidades del individuo. Respecto a lo expresado, las distintas inteligencias se encuentran disponibles en la estructura cerebral solo esperan por el poder de concentración del individuo, con la finalidad de poner en práctica ciertas habilidades mediante el proceso racional. Bajo esta óptica, respecto a las conductas orientadas a la valoración de la vida a nivel individual, así como el de las organizaciones aptas para ello, Martin (2004), plantea el desarrollo humano en los últimos tiempos desde una perspectiva sistémica bajo un enfoque holístico perteneciente a las ciencias sociales.

Visto desde esta perspectiva, con respecto a la teoría de las inteligencias múltiples se han llevado a cabo importantes investigaciones, sobre las localizaciones de las funciones cerebrales para descubrir ciertas capacidades cognitivas. Así mismo, algunos expertos lograron evidenciar durante los avances desarrollados a través de la Neurociencia crear ciertas condiciones necesarias para estudiar y analizar algunas zonas del cerebro en donde podrían estar ubicados algunos espacios de cognición.

No obstante, según los postulados de Ander (2006), la teoría de Las Inteligencias Múltiples no solo aporta distintas maneras de comprender a través de la inteligencia humana también tiene como finalidad de establecer un nuevo enfoque teórico referencial, es decir, que el individuo a través de las experiencias pueda fundamentar cambios significativos mediante los procesos de enseñanza, y aprendizaje. Ahora bien, según los estudios realizados por Gardner (1999), plantea que el ser humano posee siete tipos de inteligencias como la (lingüística, lógico-matemática, creativa, musical, espacial, cinestètico-corporal, interpersonal e Intrapersonal), sin embargo, en la actualidad existen otras inteligencias como la ecológica, espiritual, afectiva, básica, ética, estratégica, motivacional, multifocal, entre otras.

En este sentido, la importancia de analizar cómo trabaja la mente del ser humano, es decir; cómo razona, cuál es el estilo de aprendizaje que posee a través de la experiencia diaria, al momento de resolver los problemas, y sus inclinaciones. Por su parte, según los planteamientos de Seijo, 
Fuenmayor, \& Barrios, (2011), la teoría de Las Inteligencias Múltiples lleva a replantear algunos aspectos importantes durante el proceso de enseñanza-aprendizaje en cuanto a la formación del individuo en el ámbito profesional o laboral en función de sus objetivos personales.

Cabe destacar que varias investigaciones realizadas por algunos expertos en Psicología asumen que es primordial establecer distintas estrategias de evaluación para el proceso de enseñanza así como el reconocimiento de que toda persona pueda expresar a través de la experiencia el desarrollo de su inteligencia pero en diferentes manifestaciones, al poner en práctica sus objetivos individuales y sociales ante la sociedad con el propósito de que el mismo sea constructor del conocimiento.

\section{Funciones de la inteligencia ética: actuación orientada a la congruencia de la conducta humana}

Al respecto, Clemenza \& Ferrer (2004), plantean que la ética juega un papel fundamental en el pensar, decir y actuar al momento de tomar decisiones, sin embargo, la misma puede proporcionar un conjunto de reglas mediante la buena conducta, en los casos concretos sean los individuos capaces de saber exactamente qué hacer para solucionar las adversidades para el logro de los objetivos sin perjudicar a nadie, motivo por el cual se convierte entonces en un acto de libertad y sentido de pertenencia para el bien común de todos en la organización. En virtud de ello, se tiene la tendencia que las personas tengan un plan global de vida que integre sus diversas orientaciones en cuestión para poseer un sólido sentido ético. Por otro lado, es importante saber cómo el individuo en sociedad cumple, en términos de compromisos, que avale una actuación congruente en la vida ciudadana.

En virtud de estas razones, según Etkin (1998), en las organizaciones se construye un sistema de valores, definidos que permite y lo deseable para sus integrantes para evitar una desviación que destruya lo armónico. En tal sentido, dichos deberes éticos no son cerrados, es decir; que son transparentes, porque funcionan en un marco complejo desde el punto de vista social, cultural y económico, entre otros; que atraviesan las organizaciones para proyectar sobre su interior una actitud congruente entre su pensar y actuar.

De esta manera, según Seijo (2010), la inteligencia en conjunto con la ética se ha convertido en una temática de discusión frecuente e importante debido a los constantes cambios que se han presentado en las organizaciones hoy en día. Sin embargo, la inteligencia ética es habilitada por la imaginación moral, la cual es capaz de crear posibilidades inéditas de actuación orientada a la congruencia de la conducta humana. Sustentando este marco de ideas, se basa en los principios, valores, que nutre y lleva a cabo la esencia del ser humano para conservar la congruencia en cuanto a su identidad en cuestión ante la sociedad. Al respecto, Belohlavek (2007) plantea que la inteligencia ética cumple distintas funciones en la conducta del individuo a saber:

\section{Generar valor hacia el medio ambiente}

De este modo, el nivel de inteligencia ética por la que se encuentre el individuo, tendrá mayor o menor predisposición natural, es decir; que dependiendo de la actitud que tenga el mismo para enfrentar cualquier situación en el medio que se desenvuelve, el nivel de su inteligencia le resulta más fácil para agregar valor al medio. Asimismo, en el extremo superior el individuo agrega valor al medio sin buscar nada a cambio para poder lograr sus objetivos que afirmen su identidad moral ante los demás.

En este sentido, en el campo espiritual esto es frecuente, en cambio en el campo material no, sustentando lo anterior, en el marco del extremo exterior el hombre busca vivir a expensas del medio donde es el "sobreviviente"; por ejemplo, un gerente en la organización, al momento de solucionar o tomar alguna decisión, este deberá ser un potenciador para crear un ambiente de paz en la organización logrando sus objetivos con la finalidad de no tener miedo a elegir mejores opciones para resolver un propósito y así sentir orgullo por lo que hace. 


\section{Influencia del individuo en el medio que actúa}

No obstante, cuando más influye el individuo en el medio en donde se desenvuelve, es decir, en cuanto a sus buenas costumbres, o actitud congruente entre su pensar, decir y actuar, mayor es el nivel y la cantidad de individuos que lo siguen. Por lo tanto, para agregar valor al medio la persona se basa en la funcionalidad de su identidad e inteligencia ética. En efecto, este tipo de inteligencia en el individuo influye en las competencias emocionales del saber actuar, como las competencias éticas, y el actuar basado en valores. En este orden de ideas, "cuando se hace referencia a la influencia del individuo en el medio que actúa, se refiere tanto a los aspectos desde el punto de vista individual como a los grupales, donde la inteligencia ética funciona adecuadamente cuando el individuo logra los objetivos que afirman su identidad, sintiendo un orgullo especial por lo que es, hace, y siente vergüenza cuando comete una falla”. (Belohlavek, 2007, p. 11).

\section{Manejo del tiempo interno y externo que dispone el individuo}

Por su parte, según González, Marín, \& González, (2007) cuanto más tiempo necesite un individuo para interactuar en el medio más inmediatista necesita ser su actitud ética. De esta manera, mientras el individuo busque transformar conocimientos en fundamentos al procurar valor agregado, más largo será el tiempo interno que dispone el mismo. Sin embargo, esta función tiene como objetivo maximizar el valor agregado al medio, independientemente de su ética. De esta forma, se requiere para su utilización una actitud de desprendimiento muy grande, ya que su inteligencia parte del supuesto que todo tiene razón en sí, variando solo la funcionalidad de esta razón y así evitar la desesperación al momento de solucionar problemas.

\section{Capacidad estratégica del individuo}

Según Belohlavek (2007) , "La capacidad estratégica de un individuo depende de la capacidad de agregar valor, de su influencia en el medio, y el manejo del tiempo. No obstante, un individuo socialmente inteligente en un contexto organizacional podrá desarrollar su potencialidad actuando por su propia elección y no como personaje en cumplimiento de las demandas sociales de su rol”. (p.12). Sustentando este marco de ideas y a modo de ejemplo, los líderes y las organizaciones tienen la capacidad de analizar si los miembros son o no éticos, en la educación y en el ejercicio profesional que estas ofertan, donde algunas veces se observan que no poseen un conjunto de reglas las cuales puedan seguir al pie de la letra en situaciones específicas, sino por el contrario deben tener legitimidad de actuación.

\section{Liderazgo ético: centrado en la confianza, credibilidad, y coherencia}

Las organizaciones como grupo humano responsables están capacitadas para generar riquezas al momento de responder a las necesidades sociales y económicas, con la finalidad de evaluar las dimensiones de la productividad para el bienestar común de la misma. Asimismo, en la medida en que se plantee como una empresa congruente en su comportamiento a través del marco de unos valores éticos compartidos. Sin embargo, los valores y principios son escalas importantes donde las personas y organizaciones, otorgan factores propios del entorno que permite determinar las formas de ver la vida, es decir, que son influenciados por las acciones y los juicios de la gente en los diferentes escenarios en donde actúan.

Según los planteamientos de Perdomo \& Cardona, (2010)para orientar un proyecto de nuevos desafíos en la organización, donde se toman en cuenta las metas alcanzables que va a orientar la definición de la tarea de todos, es necesario promover una memoria innovadora como integración armónica, desde un punto de vista institucional y personal.

Por otra parte, Yuki (2008) asume que el liderazgo es un constructo ambiguo que parece incluir varios elementos que resulta útil para hacer una distinción entre la ética de un líder individual. Sin embargo, algunos criterios que podrían ser relevantes para juzgar a los líderes individuales, 
al incluir los valores humanos, la etapa del desarrollo moral, intenciones conscientes, libertad de elección, la dignidad humana, entre otras; para el recurso a una conducta ética congruente o inmoral, y los tipos de influencia que utiliza el individuo en la sociedad.

De esta manera, una dificultad al momento de evaluar la identidad moral de los líderes individuales en la organización es la subjetividad inherente para determinar los criterios que se van a utilizar y su importancia relativa. En tal sentido, el liderazgo basado en valores se ejerce hacia las personas, por tanto, a partir de esta premisa las personas son la verdadera clave de la empresa; motivo por el cual el liderazgo ético pasa por alto este principio pero se sostiene de tres criterios que resultan indispensables para el buen comportamiento del líder como la confianza, credibilidad y coherencia. Por tanto, los tres conceptos están unidos entre sí, cada uno se entrelaza con los otros dos. Según los postulados de Handy, (2005) el liderazgo ético exige tener presente algunos valores como:

\section{Confianza}

Asimismo, la confianza juega un papel fundamental en el líder para entrar en lo desconocido y persuadir o motivar a otros en la organización, para que vayan donde nadie ha ido antes. Sin embargo, las empresas de hoy necesitan innovar y arriesgarse para poder alcanzar los objetivos propuestos, pues estamos en un ambiente competitivo y riesgoso en la que todos estamos luchando por ser los primeros. No obstante, el líder para ser coherente con lo que proyecta ante los demás debe transmitir lealtad porque a través de esta, la confianza juega un papel fundamental.

\section{Pasión por el trabajo}

No obstante, cuando el líder siente pasión por el trabajo proporciona una energía o motivación que ayuda a impulsar a la organización para que sirva de ejemplo para otros; pero esto también tiene gran relevancia con lo opuesto, y la conciencia que existen en creencias. En efecto, en las organizaciones de hoy en día deben existir líderes apasionados, exploradores, liberales, con deseos de experimentar nuevos proyectos, y conocer otros ideales para seguir creciendo y mejorar el bienestar todos en la organización. Es así como algunos líderes hacen cambiar de opinión a las personas y las impulsan alejarse de las preocupaciones egoístas hasta llegar al servicio del bien común, esto requiere de actitud para saber orientar a los demás.

\section{Dimensiones del liderazgo ético: atributos personales del líder en la organización}

Desde un enfoque científico-social los observadores han creído durante mucho tiempo que los rasgos personales tales como la integridad ética, el carácter, el altruismo, motivación colectiva, y la conciencia ética, son indispensables para un liderazgo ético confiable en la organización. Por su parte, los líderes son considerados como personas justas, con unos principios éticos que los ayudan a tomar decisiones para el bienestar común de la gente.

En este marco de ideas, el liderazgo ético genera una relación positiva con la confianza afectiva en el líder y una relación negativa con la supervisión abusiva, pero no está relacionada con la similitud percibida entre el líder y subordinado. Según los planteamientos de Treviño \& Brown, (2006) lo más importante, son las percepciones de los subordinados respecto a la influencia del liderazgo ético en la organización donde se percibe la satisfacción con el líder, garantizando esfuerzo extra en el trabajo por medio de la voluntad para informar sobre los problemas de gestión.

Asimismo, el liderazgo ético puede ser más importante que nunca en esta nueva era en las organizaciones donde hay más empleados trabajando de forma independiente, fuera del lugar, y sin supervisión directa. No obstante, los valores éticos en las organizaciones son el pegamento que puede mantener las cosas juntas y equitativas para la armonía de la organización con el propósito de estimular a las personas hacia la producción para alcanzar los resultados sin procedentes. De acuerdo con los planteamientos de Brown \& Mitchell (2005) asumen que a nivel mundial se llevó a cabo una revisión exhaustiva 
en varias empresas donde se identificaron cinco dimensiones claves que parecen caracterizar el liderazgo ético, estos atributos, se analizan con mayor profundidad de la siguiente manera:

\section{Carácter}

Se refiere a las inclinaciones y virtudes que proporcionan la ética o fundamento moral de la conducta, según Petrick \& Scherer (1997) plantean que los líderes de carácter se manifiestan en actos de humildad, lealtad, virtud en la generosidad y en el perdón. Así mismo, la primera cualidad de todo líder es el carácter que se hace día a día en cada actuación que se manifiesta ante cualquier adversidad.

Sin embargo, los líderes deben confiar en su voz interior, la brújula interior que les señala la dirección ética, aborda el tema de liderazgo ético de "carácter" a largo plazo, que se entiende no como de carácter individual, sino de los principios que gobiernan la efectividad humana. Por su parte, Jordán, Brown, \& Treviño, (2010) (citados por Brown \& Mitchell, 2005), plantea que la conducta ética es el resultado de las propias disposiciones personales, y no el resultado de la experiencia de aprendizaje.

\section{Integridad ética}

Hoy en día uno de los rasgos más citados según sea necesario a fin de ejercer un liderazgo ético es la integridad. Se indica, así mismo, que los líderes sin integridad sólo están poniendo en un acto, una vez que los valores se han acordado y en su lugar, el director general debe ponerlos en práctica. En tal sentido, según Minkes, Small, \& Chatterjee, (1999) los líderes que demuestran la integridad ética son honestos consigo mismos y con los demás, aprenden de los errores y están constantemente en el proceso de auto-mejoramiento. Para tal efecto, el enfoque basado en la integridad de la gestión ética combina la obediencia de la ley con un énfasis en la responsabilidad de la gestión de un comportamiento ético.

$\mathrm{Al}$ respecto, la integridad ética se refiere al conjunto de estándares de honestidad y conciencia moral en que se basa en la conducta ética de una persona hacia los demás. Así, sin integridad nos traicionamos a nosotros mismos, a los demás y se destruiría la armonía de todo lo que iniciamos. Según Treviño \& Brown (2006) plantean que la integridad ética es una característica primordial para un liderazgo ético eficaz en la organización. Sin embargo, según Cortina (2008) el directivo ha de ser consciente que dependiendo de la actitud que tenga para hacer las cosas transmite un ejemplo a seguir para los integrantes de la organización.

\section{El altruismo}

De acuerdo con los planteamientos de Resick, Hanges, Dickson, \& Mitchelson, (2006), (citados por Treviño y Brown, 2006) plantean que el altruismo designa "Procurar el bien ajeno aún a costa del bien propio”, para ayudar a otros sin exigir nada a cambio, por tanto, el término altruismo se refiere a la preocupación desinteresada por los otros, lo opuesto al egoísmo. Asimismo, "suelen existir diferentes puntos de vista sobre el significado y alcance del altruismo. En síntesis, la conducta solidaria de un líder es cuando un individuo espera de alguna forma ser recompensado por el otro o por algún otro miembro del grupo para satisfacer las necesidades del mismo. Por tanto, el comportamiento altruista no solo se aplica a las personas sino también a los animales”. (p.52)

\section{Motivación colectiva}

El líder con valores éticos se orienta al desarrollo de la persona para satisfacer las necesidades de la sociedad, es decir, que es capaz de entusiasmar con sus ideas, metas, o nuevos proyectos, buscando la adhesión de quienes lo acompañan en esta aventura. Sin embargo, según Treviño \& Brown (2006) el líder ha de ser capaz de motivar a los demás y darle sentido a lo que está realizando al mostrar por ejemplo, la inserción del proyecto en un contexto más amplio, les dice cómo éste contribuye al desarrollo del país, es decir; que el líder debe ser humilde en todo lo que proponga para el beneficio de todos. 


\section{Conciencia ética}

Es la capacidad de percibir y ser sensible a los temas relevantes que merecen tener una consideración moral en la toma de decisiones que tienen un significativo impacto en los demás. De este modo, Jordán, Brown, \& Treviño, (2010) señalan que la conciencia ética se aplica tanto en las acciones o decisiones como a los procesos utilizados para alcanzarlos. En efecto, es importante que los líderes sean poseedores de la razón de buena voluntad y de una gran capacidad para colaborar con otros seres humanos.

Ante esta situación, según Resick et al (2006) quien ejerza funciones de líder debe tener conciencia ética para asumir y defender sus valores debido a su condición de ciudadano y al mismo tiempo ante la organización, debe ser cuidadoso, coherente, entre lo que dice y hace; es decir, el líder debe modelar en forma precisa dentro de la empresa velando para que se cumplan las políticas, normas, que a su vez satisfagan las necesidades de la comunidad; de esta manera, genera credibilidad y confianza, ante todo el personal que lo rodea.

\section{Inteligencias Múltiples y Liderazgo ético: factores claves en las entidades financieras}

El cambio que caracteriza el entorno económico actual plantea importantes desafíos en las entidades financieras, y por extensión al estar sometidas a las tensiones del mercado. De esta manera, el empleo productivo de recursos se asocia a la importancia que tiene la vivencia, convivencia, y en última instancia la sobrevivencia de la empresa. Según Perdomo \& Cardona (2010), todos los miembros de la organización deben ser tratados como clientes para poder satisfacer sus necesidades; recordando que sin la colaboración de los trabajadores con los objetivos de la empresa y el proyecto del líder será difícil de cumplir.

En este sentido, los compromisos hacia la búsqueda para contribuir en cuanto a la valoración, y reconocimiento hacia un mejor bienestar para el talento humano en las organizaciones surgen las inteligencias múltiples como una condición inherente para descubrir distintas capacidades cognitivas de los trabajadores en las empresas de hoy, y el liderazgo ético podría ser visto como una plataforma que le sirva a la organización de mecanismo para asumir de manera integral este indispensable propósito con la finalidad de que los gerentes puedan evitar comportamientos no éticos que afectan de una manera u otra al ser humano.

Es importante destacar que el término inteligencia se considera muy importante hoy en día, no solo en el ámbito educativo sino también a nivel organizacional, según Fernández (2004), poner en práctica la inteligencia al turbulento y riesgoso mundo empresarial, se traduce como gestión del conocimiento lo cual viene a proclamar al mismo, como una capacidad estratégica para resolver problemas. A modo de reflexión, se debe tener en claro que actuar bien, con elevado rendimiento, exige más conocimiento, habilidades sociales, determinadas creencias, actitudes adecuadas, fortalezas de carácter, motivo por el cual esto es actuar estratégicamente y con inteligencia.

Visto de esta forma, según Perdomo \& Cardona (2010), las organizaciones deben saber controlar, su gestión con inteligencia para poder sobrevivir organizacionalmente, es decir; a través de una interacción honesta que enfatice lo emocional, racional, ético, espiritual, en las relaciones interpersonales y en el trabajo de equipo, siendo estos los factores básicos de integración para mejorar la tendencia del mundo empresarial actual. Por ello, se hace necesario que las mismas se manifiesten mediante una plataforma ética que conlleve a su talento humano con el fin de aprender a valorar sus potencialidades y cultivar sus capacidades.

En este sentido, contrastando lo anterior con los planteamientos teóricos de Treviño \& Brown (2006), asumen que los lideres éticos se deben adaptar a los cambios de manera eficiente, eficaz, con el propósito de movilizar los valores éticos centrales y el compromiso de estimular a las personas hacia la producción de resultados sin procedentes. 
Asimismo, en las organizaciones, y en especial en las entidades financieras, dejan mucho que decir respecto a los valores éticos que deben poner en práctica el líder de hoy, es decir; una de las causas es que muchos gerentes ignoran la importancia de comprender que sin principios y valores no se podría convivir en un ambiente armónico en una empresa o sociedad de personas, donde el liderazgo ético exige conjugar el nosotros a través de la honestidad, y solidaridad. No obstante, en las actuales circunstancias ha surgido una creciente incertidumbre de saber si los líderes ayudan a estabilizar la influencia de los valores en la organización, a través de la ejemplaridad hacia todos los niveles para mantener un orden ético empresarial valido para todos.

Sustentando lo anterior, según Treviño \& Brown (2006), las organizaciones deben ser dirigidas por líderes con visión estratégica para construir la empresa del futuro a través de la siembra de unos valores éticos, con la finalidad de fortalecer una cultura organizacional orientada al éxito por medio del trabajo en equipo, en pocas palabras; se trata de asumir el compromiso al momento de integrar y ganar las voluntades de quienes están involucrados directamente con la empresa. Por tanto, el líder debe ser el primero en exhibir y practicar los valores.

Dentro de este marco de ideas, se requiere entonces, de gerentes con una elevada formación ética que sean capaces para construir condiciones socialmente aceptables, asumiendo una filosofía empresarial basada en un enfoque de aprendizaje continuo de adaptación axiológica, es decir, que la ética en el liderazgo, como código enfocado a orientar la conexión de inteligencia con el ethos de la organización pueda contribuir al bienestar común para la empresa, con un personal altamente capacitado y comprometido que garantice el éxito organizacional a futuro.

A modo de reflexión, las entidades financieras deben saber controlar su gestión con inteligencia para poder sobrevivir organizacionalmente, es decir, a través de una interacción honesta que enfatice lo emocional, racional, ético, espiritual, en las relaciones interpersonales y en el trabajo de equipo, siendo estos los factores básicos de integración para mejorar la tendencia del mundo empresarial actual con la finalidad de ayudar a incidir positivamente en la toma de decisiones, entre la más importante, la financiera, que sirve para el financiamiento al momento de obtener inversiones y desarrollar las actividades institucionales.

En síntesis, se puede deducir de la importancia que tiene una gerencia proactiva, que considere mayor interés por las expectativas de cada uno de los empleados, e incentivarlos a su crecimiento profesional en relación a las competencias exigidas en el mercado. Por tanto, a través de la inteligencia empresarial, el buen liderazgo y la ética, las empresas aseguran su capacidad para el saber hacer, así como para hacer más y mejor con lo que se tiene, para mejorar su relación e interacción con el entorno.

\section{CONCLUSIÓN}

Ser inteligente en el ámbito empresarial, y en especial en las organizaciones venezolanas, es saber cómo solucionar los problemas, manejar los conocimientos del capital humano de una organización, para estimularlos a poner en práctica su potencial creativo e innovador, ofreciendo nuevas propuestas no solo a las situaciones que se estén presentando sino para desarrollar nuevos proyectos de acuerdo a las exigencias en la actual sociedad.

Asimismo, las organizaciones necesitan de líderes que tengan como finalidad, satisfacer ciertas necesidades sociales de los consumidores a través de los servicios de alta calidad, es decir, se deben comprometer a promover el bienestar organizacional e individual de sus empresas, al transferir sus conocimientos, experiencias, a los sectores emergentes, participando activamente en la formulación de políticas, económicas, sociales, a favor del bien común con el propósito de cubrir con éxito a las exigencias de los mercados en un mundo cambiante como el de hoy en día.

En síntesis, ello implica un estilo gerencial estratégico, proactivo, visionario e inteligente, el cual permita al talento humano exteriorizar a través de cada individuo el potencial innovador que 
las condiciones de la sociedad actual exige para garantizar la transparencia de la misma, motivo por el cual estos son esenciales para el buen desenvolvimiento ético de cualquier empresa.

\section{REFERENCIAS BIBLIOGRÁFICAS}

Ander, E. (2006). Claves para introducirse en el estudio de las inteligencias múltiples. Editorial Homosapiens. Santa Fe - Argentina.

Belohlavek, P. (2007). Introducción a la inteligencia ética. Editorial. Tagle Group Book- New York.

Beauport, E. \& Díaz, A. (2008). Las tres caras de la mente. Orquesta tu energía, las múltiples inteligencias de tu cerebro triuno. 1era. Edición. Editorial - Galac, S.A.

Brown, M. \& Mitchell, M. (2005) Liderazgo ético y no ético: Exploración de nuevas vías para la investigación futuras. Revista Business Ethics Quarterly, vol 24, no. 4, pp. 583-616

Cortina, A. (2008). Ética de la Empresa. Octava Edición. Madrid, España. Editorial - Trotta, S.A.

Clemenza, C. \& Ferrer, J. (2004). Ética de la gestión pública: Plataforma de sostenibilidad suscrita por la sociedad civil, clave en la lucha anticorrupción en: XI Congreso internacional del CLAD sobre reforma del estado y la administración pública. Madrid, España. Noviembre - 2004.

Etkin. J. (1998). El potencial ético de las organizaciones. Las formas de integrar la eficacia con los valores sociales. Seminario internacional: Los desafíos éticos (Documento en lìnea). Disponible en: http://www.iadb.org/etica/documentos/ ar_2etk_etico.doc_Consultada en Junio - 2012.

Fernández, O. (2004). Inteligencia Solidaria. Ediciones. Ricardo Vergara. Buenos Aires.

Gardner, H. (1999). La inteligencia revisitada. Las inteligencias múltiples en el siglo XXI, Cuarta Edición, Paidós - Barcelona.

González, E. Marín, M. \&. González, M. (2007). La inteligencia ética: alternativa para el logro de organizaciones educativas socialmente responsables. Multiciencias, Vol, 7. Número 002. Consultado el 14 de abril del 2012 Disponible en: http://redalyc. uaemex.mx_Universidad del Zulia. Punto Fijo - Venezuela.

Handy C. (2005). La organización, por dentro: por qué las personas y las organizaciones se comportan como lo hacen. Editorial - Deusto, Argentina.

Jordán, J., Brown, M. Treviño, L. \& Finkelstein, S. (2011). Alguien a quien admirar: Ejecutivo-seguidor razonamiento ético y la percepción de un liderazgo ético. Disponible en: http:// leeds faculty.colorado.edu/mcgrawp/PDF/Jordan. Brown.Trevino.Finkelstein.forthcoming.pdfel $27 \mathrm{de}$ Marzo-2012.
Martin, V. (2004). La inteligencia ética en organizaciones públicas. IX Congreso Internacional del CLAD sobre la Reforma del Estado y de la Administración Pública, Madrid, España, 5 de Noviembre - 2004 Disponible en http://www.iij.derecho.ucr.ac.cr/ archivos/documentacion/inv \% 20otras \% 20entidades/CLAD/CLAD\%20IX/documentos/martinfi.pdf Artículo [En Línea] citado en Abril - 2012.

Minkes, A. Small, M. \& Chatterjee, R. (1999). Liderazgo y ética en los negocios: Implicaciones para la gestión. Journal of Business Ethics, 20(4), 327-335. Disponible en http://link.springer.com/ar ticle/10.1023\%2FA \%3A1005741524800 Consultado en Junio-2012.

Perdomo, \& Cardona. (2010). Liderazgo ètico en la universidad valle del momboy. Revista electrónica facultad de ingeniería. Volumen 4. Edición No. 2. Venezuela.

Petrick, J. \& Scherer. R. (1997) Ética de gestión: Integridad en el Trabajo. Serie Sage en Ética Empresarial, Thousand Oaks, CA. Volumen 18. N.1. Disponible en : http://www.wright.edu/studentorgs/sie/docs/faculty_resources/erm_3.pdf Consultado el 22 de Marzo - 2012.

Romaña, G. (2010). Inteligencia ètica y negociación en entidades financieras binacionales. Tesis Doctoral. Publicada. Universidad Privada Rafael Belloso Chacìn. Consultada en Agosto-2012.

Resick, C. Hanges, P. Dickson, M. \& Mitchelson, J. (2006) Un examen transcultural de la aprobación de un liderazgo ético. Journal of Business Ethics, Disponible en: http://www.bsos.umd.edu/psyc/ hanges / Resick \% 20Hanges \% 20Dickson \% 20 Mitchelson \% 202006\% 20Cross \% 20cultural \% 20 examination \% 20of \% 20ethical \% 20leadership.pdf Consultado el 12 de Abril - 2012.

Seijo, C. Fuenmayor, R. \& Barrios, L. (2011). Las inteligencias múltiples: una aproximación hacia la inteligencia ética como característica congruente de la actuación humana. Revista Formación Gerencial. Disponible en: http://dialnet.unirioja. es $/$ servlet/articulo? codigo $=3751750$ Consultado en Marzo -2012

Seijo, C. (2010). La gerencia en la sociedad: un camino para la construcción de organizaciones futuras con rostro humano. 1ra. Edición. Venezuela. EditorialAstro Data S.A.

Treviño \& Brown. (2006). El liderazgo ético: Una revisión y direcciones futuras. Disponible en http:// www.publicpolicy.umd.edu/MLI/Leadership/ Ethical.\%20Leadership.pdf Sam and Irene Black School of Business. The Pennsylvania State University-Erie. Consultado Marzo - 2012.

Yuki, G. (2008). Liderazgo en las organizaciones. $6^{\text {a }}$ Edición. Editorial Pearson- Educación, S.A. Madrid. España. 